\title{
Smart sensors
}

\author{
Khalil Najafi \\ Center for Integrated Sensors and Circuits, Department of Electrical Engineering \\ and Computer Science, University of Michigan, Ann Arbor, MI 48109-2122, USA
}

Received 14 February 1991, accepted for publication 1 Aprii 1991

\begin{abstract}
This paper is a state-of-the-art review of solid-state integrated and smart sensors. Smart sensors are defined as sensors that provide analog signal processing of signals recorded by sensors, digital representation of the analog signal, address and data transfer through a bidirectional digital bus and manipulation and computation of the sensor-derived data. In this paper the overall architecture and functions of circuit blocks necessary for smart sensors are presented and discussed. Circuit fabrication technologies are briefly discussed and cMos technology is found to be ideally suited for many sensor applications. The challenges and techniques for the packaging of smart sensors are briefly reviewed and several specific examples of solid-state integrated and smart sensors are presented. It is believed that smart sensors will be needed in future closed-loop instrumentation and that control systems will be required in many application areas, including automotive, health care, industrial processing and consumer electronics.
\end{abstract}

\section{Background}

The term 'smart sensor' has been used by various researchers in a number of different contexts, ranging from sensors incorporating a few active devices to provide a more reliable interface to the sensor to enhance the quality of the sensed signal, to integrated sensors incorporating a sophisticated electronic circuit block including both digital and analog circuitry that helps transform the sensor from a passive component into a 'smart' periphery of a control/instrumentation system. However, over the past few years, a rather broad consensus has been achieved whereby a smart sensor is defined as one that is capable of: (i) providing a digital output; (ii) communicating through a bidirectional digital bus; (iii) being accessed through a specific address; and (iv) executing commands and logical functions $[1,2]$. In the context of this paper, the smart sensor is defined as one that should possess these features. In addition, it is also desirable for the smart sensor to perform such functions as compensation of secondary parameters (e.g. temperature), failure prevention and detection, self-testing, autocalibration and various computationally intensive operations. The development of such sensors will greatly enhance the capabilities of many control and instrumentation systems that are nowadays lacking in their interface with the external world.

Figure 1 shows the elements of a typical electronic control system. Sensors provide analog information on the system being monitored through signal conditioning circuits to a microprocessor-based controller. The processor interprets the information, makes appropriate decisions (perhaps in conjunction with higher level control), and implements those decisions via the actuators. Sensors currently represent the weakest link in the development of most next-generation instrumentation and control systems. Where sensors exist at all, they are frequently unreliable, rarely attain an accuracy of 8 bits and may cost more than the processor. This situation is especially true in biomedicine, where the problems are particularly difficult. Only in the past few years has this situation begun to change.

Sensors have progressed through a number of identifiable generations, as shown in figure 2. Firstgeneration devices had little, if any, electronics associated with them, while second-generation sensors were part of purely analog systems with virtually all of the electronics remote from the sensor. By the third generation, where

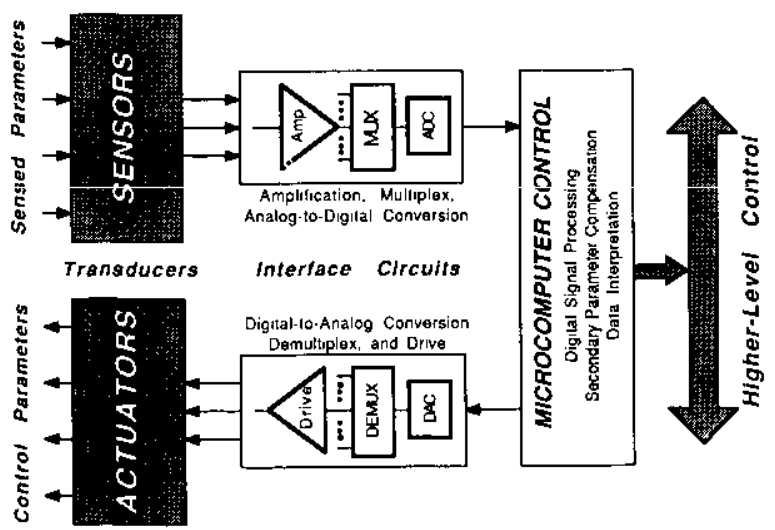

Figure 1. A typical electronic control system [22]. 

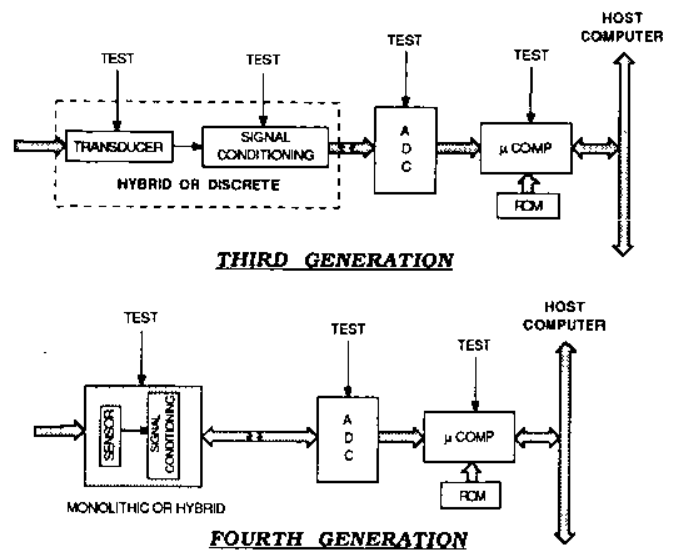

FOURTH GENERATION

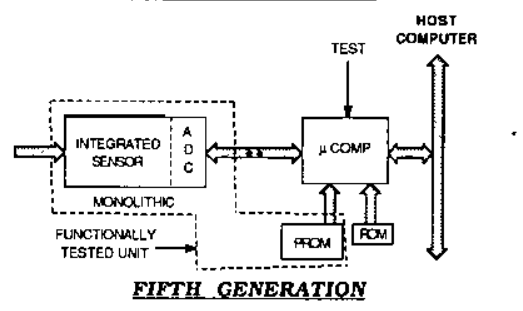

Figure 2. Evolution of solid-state sensors. (Reprinted courtesy of IEEE [21].)

the majority of the systems currently reside, at least the first stage of amplification occured in the sensor module or on the sensor chip itself. Thus, the output from these systems is a high-level analog signal, encoded either as a voltage amplitude or as a variable pulse rate. This signal is digitized remotely and then processed by a microcomputer. Many automotive sensing systems fit into this category. We are now evolving into the fourthgeneration sensors, where more analog and digital electronics are on-chip, making the sensor addressable and in some cases self-testing, with two-way communication between the sensor and the host microcomputer. Some large-area visible imaging devices [3] and pressure sensors $[4,5]$ represent fourth-generation components.

On the horizon are fifth-generation sensors, in which data conversion is accomplished on the sensor (or, at least, in the sensor module) so that the bidirectional communication link with the microcomputer is digital. These devices will likely be digitally compensated using field-programmable read-only memories (PROMs) to achieve 12-bit accuracy over a wide dynamic range. Such accuracies are not available in sensors today. From a system viewpoint, the standardization of communication protocols and formats is badly needed for this fifth generation of devices. Figure 3 shows the block diagram of a generic fifth-generation VLSI sensor. The device is addressable, self-testing and communicates over a bidirectional digital bus. It can measure the outputs of a variety of sensors, use some of these outputs for secondary parameter compensation, perform digital signal processing tasks and retain only the useful and required signals and then communicate the acquired signals with the higher level computer. For example, a gas sensor might sense gas pressure, temperature, flow and include an array of gas sensors to improve selectivity and

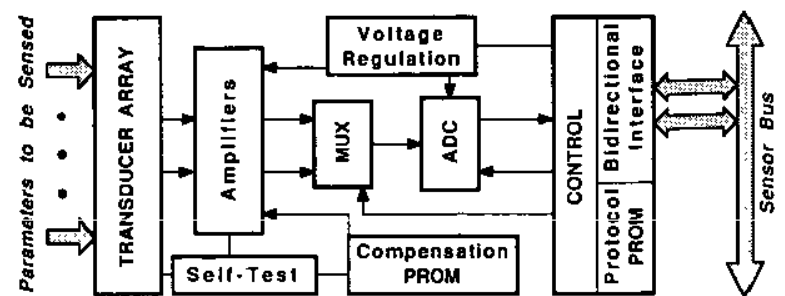

Figure 3. Block diagram of a generic fifth-generation VLS1 sensor [22].

sensitivity. The required device counts for such a sensor, estimated in the range of ten thousand, are still low enough that the circuitry would occupy no more than a few square millimeters and would not be a major cost factor as most of the cost would be in testing and packaging.

Based on the evolution of both sensor technologies and electronic systems, it is evident that solid-state sensors will continue to incorporate more sophisticated electronic circuits. The integration of high-performance solid-state sensors and sophisticated signal processing and control circuitry into a smart sensor module serves a dual purpose: it allows the sensor to become a much more versatile component in terms of its sensing function and accuracy; and will enable the smart sensor to become an active and compatible component of an overall 'sensing system'. It is believed that unless these systems are developed and demonstrated to be capable of surpassing the performance of many of the existing sensors, solid-state sensors will not be fully utilized in terms of their capabilities and features. The development of such systems will naturally require the development of individual components and circuit blocks that make up the overall system.

\section{Overall system architecture and functions}

Implementation of smart sensors for many applications requires the inclusion of three main circuit blocks. These are signal processing, digital control and manipulation, and external communication and bus interaction. Each of these areas are discussed in more detail below.

\subsection{Signal processing}

The signals recorded by many sensors are typically low in amplitude, and the sensor interface usually exhibits a high impedance at frequencies of interest. Integration of interface electronics and signal processing circuitry at the sensor site (monolithic or hybrid) serves a number of functions, including signal amplification, impedance transformation, signal filtering and buffering, and multiplexing.

Amplification of sensor signals remains one of the most important functions in many applications for which the amplitude of the signal is typically low. Amplification of these signals at the sensor site before transmission to the outside world not only enhances the overall signal-to- 
noise ratio thus reducing the effects of noise from the environment, but also allows for the full utilization of the dynamic range of an analog-digital converter for those sensors that incorporate an ADC in the sensor module. For many integrated sensors this amplification can be achieved by using mos and bipolar amplifiers that require only a minimal amount of circuitry with nominal gain, bandwidth and performance specifications. CMOS amplifiers are perhaps the most suitable since they provide high gain and high input impedance through a relatively simple and compact circuit and are readily compatible with integration of high-density digital circuitry on the same chip [6]. CMOs amplifiers are now easily capable of prividing open-loop gains of $90 \mathrm{~dB}$, are fast, can be compensated for their offset using various techniques involving charge storage and cancellation, and consume little power and area. These amplifiers are 3-5 times smaller than bipolar amplifiers, making it possible to include tens of amplifiers per-channel on a single chip. In addition, the input impedance of CMOs amplifiers is very high, which makes them ideal for sensor applications. Although mos amplifiers have typically had larger noise levels than their bipolar counterparts, various circuit and fabrication techniques are used to minimize the noise in applications in which the noise level of the interface electronics determines the resolution of the sensing system [7]. More sophisticated amplifiers incorporate programmable gain capability to maximize signal-to-noise ratio and optimize the amplifier output range for analog-digital converters. Many integrated sensors typically incorporate the amplifier on-chip, especially those designed for biomedical applications for which small size and signal amplification are two of the most important requirements.

In addition to signal amplification, impedance transformation is also often required for both resistive and capacitive sensors. A low impedance output from the sensor is required not only to ensure maximum signal transfer to the next stage but also to drive output leads and reduce the susceptibility of the sensed signal to environmental noise. Transconductance amplifiers implemented in a typical cMos operational amplifier are best suited for this purpose. Often the amplification and impedance transformation can be achieved simultaneously in such circuits. For other applications where gain is not immediately necessary on the sensor chip, a simple buffer circuit can be integrated. This is particularly important for capacitive-type circuits.

Signal filtering can also be incorporated in these amplifiers. For multiplexed multisensor systems, filtering of the signal is often required to prevent such problems as aliasing that can introduce high frequency noise into the signal passband, thus overriding the low-amplitude sensed signal. Signal filtering can also improve the signal-noise ratio of the sensor as it filters the out-ofband high frequency noise that may be introduced by the electronic devices themselves. A variety of low-pass bandpass and high-pass filter circuits are available using switched-capacitor circuit techniques or more conventional resistồ-câpacitôr techñiques.
Reducing the number of output leads is another important function for a majority of sensors. Data multiplexing can not only reduce the amount of circuitry required for the sensor (circuit blocks such as the ADC can be shared among several sensors), but also reduces the number of external leads by either multiplexing several sensed signals onto a single output lead, or by superimposing clock and control signals over the power lines [8]. Low-noise analog multiplexing is an especially important function in sensing arrays and systems that require the simultaneous measurement of many signals in order to accurately measure the parameter of interest. In addition, reducing the number of leads that are interconnected to a sensor package is very important in simplifying the packaging, reducing the cost and improving the longterm reliability of the system in which the sensor is integrated. In automotive applications of sensors this has been found to be of paramount importance and it is expected that almost all sensors fabricated for this area will have particular attention paid to lead reduction [9].

In addition to these primary functions, the interface signal processing circuitry is increasingly used to perform functions such as self-testing of the analog circuit blocks and offset cancellation that will permit full utilization of the dynamic range of the ADC [10]. It should be emphasized once again that signal amplification, filtering, and multiplexing of several data channels onto a single data line also reduce packaging problems and inherently improve the reliability of the device by enhancing the signal-noise ratio and by eliminating the need for too many external leads.

Two points should be mentioned with regard to the sensor interface circuitry. First, although the design and layout of many of these circuit blocks is still custom, the progress made in the last few years in the computer aided design (CAD) of many analog integrated circuits and the availability of many cell designs for analog functions have greatly simplified the implementation of many of the functions discussed above. Silicon compilers are now available for a variety of analog functions, which relieve the sensor designer from performing detailed circuit and layout designs [11]. Many of these CAD tools have reduced design time and increased design reliability. Therefore, it is now possible to design a semi-custom analog integrated circuit for a particular sensor and fully test its functionality without committing the design to silicon. The integration of many of these circuits with the sensor has become less complicated as integrated sensors have evolved towards greater compatibility with integrated circuit fabrication processes, as will be discussed later. Second, with regard to fabrication and process compatibility, it is noted that some sensors, such as photodiodes and piezoresistive pressure sensors, rely on the operation of an electronic device anyway and incorporating additional circuitry into the existing process does not greatly complicate the overall fabrication process and cost. Other sensors, however, are fabricated in such a way that the introduction of any electronics is more involved. For example, for capacitive pressure sensors, the addition of on-chip circuititry complicates the 
process to a larger degree than for a piezoresistive pressure sensor. Therefore, the question of whether circuitry should be integrated on-chip becomes not only dependent on performance but also on how complicated the final process becomes and how this complication affects the yield and cost of the final device.

\subsection{Digital control and manipulation}

One of the main requirements for smart sensors is their compatibility with digital control and microprocessorbased systems. Most high-performance sensors should provide a digital output that can be accessed through a digital bus. Once the sensor data is digitized, a variety of signal processing schemes can be used to correct for a number of errors and shortcomings. These include offset cancellation, auto-calibration, self-testing, fault detection and correction and linearity correction. While some of these functions can be achieved using analog circuits, digital signal processing techniques are somewhat easier to implement using straightforward circuit techniques. Furthermore, it may be simpler to perform some of these functions using a remote host processor than to have to implement them at the sensor site. In the following paragraphs these features and their importance in smart sensors will be discussed.

The main circuit block required before digital control and manipulation of sensor data can take place is the analog-digital converter. Analog-digital converters have seen tremendous growth and progress over the past few years. Many of the designs for ADCs today utilize switched-capacitor circuits $[12,13]$. These techniques have allowed the integration of high-performance analog functions with low-power and high-density CMOs digital circuitry, thus allowing the implementation of very highaccuracy analog-digital converters $[14,15]$. For most sensor applications, conversion rates of $10-20 \mathrm{kHz}$ at 12 bits are probably adequate and this performance level can be readily achieved in CMOs technology today, although there is a continuing need for reduced die size and power dissipation. For some applications it may be necessary to either precede the ADC with a programmable gain amplifier in order to utilize the full range of the ADC, or to employ an ADC with an accuracy higher than 12 bits in order to cover the full dynamic range of the sensor. It should be noted that for sensor applications it is important to keep the overall design of the converter as simple as possible and to keep die area and power dissipation at a minimum. Therefore, the design of a converter for sensor applications may require a different set of tradeoffs than for more general purpose applications. It may also be possible to improve overall converter accuracy by externally compensating for converter errors, in much the same way as it is performed for the sensor data. It is believed that the design of the ADC for different sensor applications will depend on the requirements for the particular application and on the complexity of the fabrication process.

After the sensor signal is digitized, a number of functions can be performed on the sensor, its interface electronics and the digitized data itself. Auto-calibration is a very desirable function for smart sensors. Most sensors should be adjusted for changes in gain and offset, usually at factory test and before packaging. The performance of many sensors is influenced strongly by these parameters, and how well these parameters can be set will determine the level of precision and accuracy achieved. In addition, the long-term stability of sensors is also affected as these two parameters can drift over time, thus affecting the accuracy of the sensor. Therefore, it is desirable to not only calibrate the sensor at factory test, but also to have the ability to auto-calibrate in the field. In order to perform auto-calibration remotely, however, a known input signal needs to be generated and the sensor should be capable of being controlled in a closedloop fashion. This can be achieved using some of the techniques developed recently for microactuators, including actuation and control of micromachined elements using electrostatic forces. These techniques are already being employed to calibrate accelerometers [16] and ultrasensitive pressure sensors [17], and it is expected that many future sensors fabricated using either bulk or surface micromachining techniques will incorporate some type of force feedback for auto-calibration and selftesting. As for calibrating the circuitry (such as the ADC), additional circuitry and complexity is required. It is possible to use either analog trim, most likely with a specific amount of charge stored on an on-chip capacitor, or digital trim, with correction parameters stored either in on-chip memory or at a central control/processing computer $[15,18,19]$. In order to avoid complicating the design of the circuitry, and in order to improve overall yield, it is believed that the entire sensor-signal path can be calibrated using the external host processor which can access the sensor PROM. In addition to personality information such as address and the type of sensor, the PROM will store information such as coefficients of polynomials that are used to digitally compensate the sensor. Digital compensation of sensor data appears to be a very attractive technique for achieving accuracies beyond the 10-bit level [18].

Sensor data compensation is thought to be one of the main advantages and features of a smart sensor. Data compensation can be used to correct for secondary parameter sensitivities (e.g. sensitivity to temperature), for non-linearity either in the sensor signal or in the analog signal path, for drift of the sensor signal over time if one can measure the amount of drift, and also for noise. Compensating any, or all, of these sources of error wil] result in a more accurate and higher-performance sensor. Although sensor data compensation can be minimized and somewhat reduced by proper design of the sensor structure, it may be easier to compensate the sensor data after it has been digitized and transmitted to the host controller. Compensation for secondary parameters (such as temperature) is best achieved in this way since in smart sensors multiple physical and environmental variables can be measured and the desired sensor data can be extracted from the combination of these sensed signals. This technique is widely used in many physical 
transducers including pressure sensors and accelerometers.

In addition to the above functions, a large number of digital signal processing (DSP) tasks can be performed by the sensor circuitry. These DSP techniques can be used to improve the sensor performance, and the added computational power to the sensor itself will make it behave more like a system component. The sensor is now able to perform logical functions and perhaps monitor its own integrity and inform the host controller in the event of a failure. The added computational power can be utilized to automatically range the amplifiers, the ADCs, and other circuit blocks so that maximum gain, sensitivity and dynamic range is achieved for many sensors at all times, thus utilizing the entire dynamic range and bandwidth of the sensor electronics.

Besides calibration and compensation, self-testing and diagnosis are other important functions required for smart sensors. The ability to self-test is a very desirable feature since it allows the host processor to determine the functionality of the sensor without having to physically remove the sensor from its environment. Self-testing can be initiated under external control, perhaps by the external host processor, to ensure that the sensor and the entire signal path are functional corresponding to a given set of specifications. Notice that self-testing is often performed to detect gross malfunction of the sensorelectronics and may not be required for exact calibration. This was described above as auto (self)-calibration.

A third issue of importance for many control and instrumentation systems is that of reliability. This is especially important in distributed sensing systems where access to a particular sensor may not be easy and it is desirable (and often required) that the sensor be extremely reliable. Smart sensors can provide a clear advantage in this area as compared with passive sensors. The simplest means of improving reliability is to add redundancy to the sensor and the circuit. Because of their small size, integrated sensors can be easily replicated on the sensor chip in order to improve the reliability of the overall sensor. Therefore, multiple sensing paths can be created and if one fails (either at factory test or in the field), it can be taken out and a 'good' sensor can be programmed in. This technique has been used for a long time in the IC industry to improve the yield of many VLSI circuits, including DRAM chips. It is also possible to replicate the on-chip circuit blocks to increase the overall yield. Such replication does not affect processing, since all of the process steps are required by a single implementation of a given circuit. The only constraint is the space required. In many sensors, particularly those made with bulk micromachining, there is a significant amount of space between the outer edge of the transducer and the outer edge of its mechanical support [20]. This is where circuits will be located whenever possible, since this area is obtained at no cost. Analog switches and pass gates can be used to select the circuit path dynamically, or a destructive trim technique (such as cutting of unneeded interconnect lines) can be applied on a one-time basis. Another approach to improving yield and reliability would be to simplify both the circuit and the sensor design. By simplifying the circuit blocks, the possibility of device failure becomes smaller. It should be noted that there exists a trade-off between the level of complexity (and therefore the level of functionality) achieved for the sensor and the overall cost and perhaps yield. The issue of reliability is certain to remain a topic of discussion for many smart sensors and the pros and cons are believed to be as widespread as the number of application areas.

\subsection{Communication and bus interaction}

As mentioned above, a smart sensor should be capable of interacting with a higher level controller that manages the overall system. Therefore, a portion of the circuitry associated with smart sensors should be dedicated to interface with the bus in order to exchange information with the controller. Two issues are of particular importance. First, each smart sensor should be capable of interfacing with the different buses and bus protocols, especially in the face of the lack of a universally accepted bus for sensing systems. Efforts are currently underway to develop such a standard bus for sensor applications that is uniquely designed to optimize functionality, speed and overall cost [21]. In order to accommodate various buses, the bus interface circuitry can be custom designed for the specific bus of interest to communicate with the controller. In order to reduce the complexity of the bus interface design and reduce the overhead for the circuit/system designer, a library of cells designed for various buses can be created and utilized at the end of the design process for different smart sensors. Second, and perhaps more important, is the communication interface and its complexity. As mentioned above, a variety of information can be exchanged between the sensor and the controller over the bus, including calibration and compensation data, addresses and personality information, measured data, and programming data initiated by the controller. In its most complex form, the communication interface should have the ability to receive and transmit information over the bus at a fairly high speed with not only the central controller, but perhaps with other sensing units in a distributed system. For other applications, on the other hand, the major requirements are reliability, low lead count and low noise. The automotive environment is one such application. Various serial buses are being developed and particular attention is being paid to reduce the number of bus lines, perhaps at the expense of speed. However, it should be noted that many sensor signals have limited bandwidth and even in the case of a multisensor system, the bus data rate may be sufficient to accommodate all sensors. For other applications where not only large numbers of sensors exist, but where these sensors are faster and are more accurate, a serial bus may not be adequate. Various parallel buses have been used and developed such as the Michigan Parallel Standard (MPS) [21].

In summary, irrespective of the particular data bus and the particular sensor application, it is evident that smart sensors should be capable of communicating with 
a host computer through a bidirectional data bus. The interface between the semi-standard signal processing and digital electronics on the sensor chip (module), and the digital bus can be formed as a last step in the overall sensor design by selecting the appropriate bus interface circuitry from a previously designed library of standard cells.

\section{Fabrication technologies}

If sensors are to be effectively integrated into a multisensor distributed environment, they have to be more versatile and should function more like a system component than a passive module. In order to achieve this and to improve the sensitivity, stability and reliability for many sensors that will have to operate in harsh environments, electronics should be incorporated in the same module as the sensor in either a monolithic or hybrid fashion. As discussed above, smart sensors need not have electronics integrated monolithically. However, as fabrication technologies for microsensors mature, more integrated smart sensors (sensors with on-chip integrated electronics) will be developed and fabricated. For these sensors, a number of important issues need to be considered, including the selection of proper sensor technology, of appropriate circuit fabrication technology and of compatible sensor-circuit fabrication processes.

The choice of the sensor technology is often forced by the required performance characteristics of the sensor. The two main sensor fabrication technologies are bulk silicon and surface micromachining [22-24], both of which can produce microsensors that are superior in many respects to their discrete counterparts. In the selection of a proper sensor on-chip circuitry, a number of requirements have to be satisfied. These include high packing density (small die area), low power dissipation, functional versatility since the on-chip circuitry requires the integration of both analog and digital electronics on the same substrate, fabrication simplicity (high yield), and drive capability. Historically, integrated circuits have been fabricated using three main technologies: (i) bipolar junction transistor (BJT); (ii) metal oxide semiconductor (MOs); and (iii) complementary mos (CMOS).

Bipolar ICs have typically been used in very highspeed and high-drive applications in which large loads need to be driven at fairly high speeds. They therefore consume large amounts of power and area. In the digital circuit arena, bipolar technologies have been mostly replaced by mos technologies due to their complicated processing and low packing density. In the analog regime, however, many bipolar circuits and functions are being used because of the inherently higher gain and speed of bipolar analog circuits and because of the higher uniformity achieved with bipolar transistors. However, bipolar technologies do not lend themselves easily to the implementation of a number of analog functions that are critical to circuits required in sensor applications. The first important requirement is the need for high input impedance interfaces for a variety of sensors, which is usually difficult to achieve with bipolar devices unless area and power consuming circuit techniques are employed. Secondly, bipolar device structures do not lend themselves easily to analog multiplexing and cannot be used as bilateral switches. To overcome some of the shortcomings of the BJT, other technologies such as integrated injection logic [25] and Bipolar-Mos (BIMOs) [26] have been developed. BIMOS technologies have become more popular as they combine the high packing density/low power dissipation of mos technology and the high drive/gain capability of bipolar devices. They do however have a more complex fabrication process which restricts their application to very high performance applications (e.g. very high-speed VLSI circuits).

Mos circuit technologies, on the other hand, offer very high packing density and low power dissipation, which makes them very suitable for many applications. In addition, their very simple fabrication process results in high yield and increased reliability. They best satisfy the requirements for integrated microsensors. MOS devices can be easily used as bilateral analog switches (or transmission gates), this being useful for making a small multiplexer. Although they have a higher $1 / f$ noise than BJT devices, the noise level is still lower than the required level for many sensor applications. The two most frequently used Mos technologies nowadays are enhancement-depletion $N$-channel MOS (ED NMOS), and CMOS. Because of the aforementioned characteristics, it is believed that CMOS is presently best suited as the circuit technology of choice in most integrated sensors and actuators. It provides a higher gain per stage than an NMOs circuit, is capable of implementing digital as well as high-performance analog circuits, has a low-power dissipation, offers good packing density and high speed and has a mature fabrication technology that is reliable and high yield. Á number of sensors have been developed which incorporate CMOS circuitry $[5,8,20]$.

Although standard CMOs technology satisfies most of the circuit requirements for integrated sensors, the implementation of a number of functions still requires bipolar devices. Some of these functions include voltage references, voltage regulators, low-voltage current sources and sinks, and precision current sources. Voltage regulation is of special interest since as integrated sensors become more autonomous in terms of the amount of circuit functions on them, it wili become increasingly desirable for them to handle all their power regulation and referencing locally. This is even more desirable for implantable biomedical sensors that operate using radiofrequency telemetry. Based on these overall requirements, a BICMOs process technology that offers moderate bipolar device performance in terms of device speed and power handling capabilities will be required for many future integrated sensors and micromechanical systems. The use of parasitic bipolar devices and modified CMOsbipolar technologies to satisfy this need is perhaps the easiest and least expensive approach.

Figure 4 shows the cross-sectional view of a standard CMOS process that utilizes lateral and parasitic bipolar transistors for circuit applications [27]. The cMos circui- 


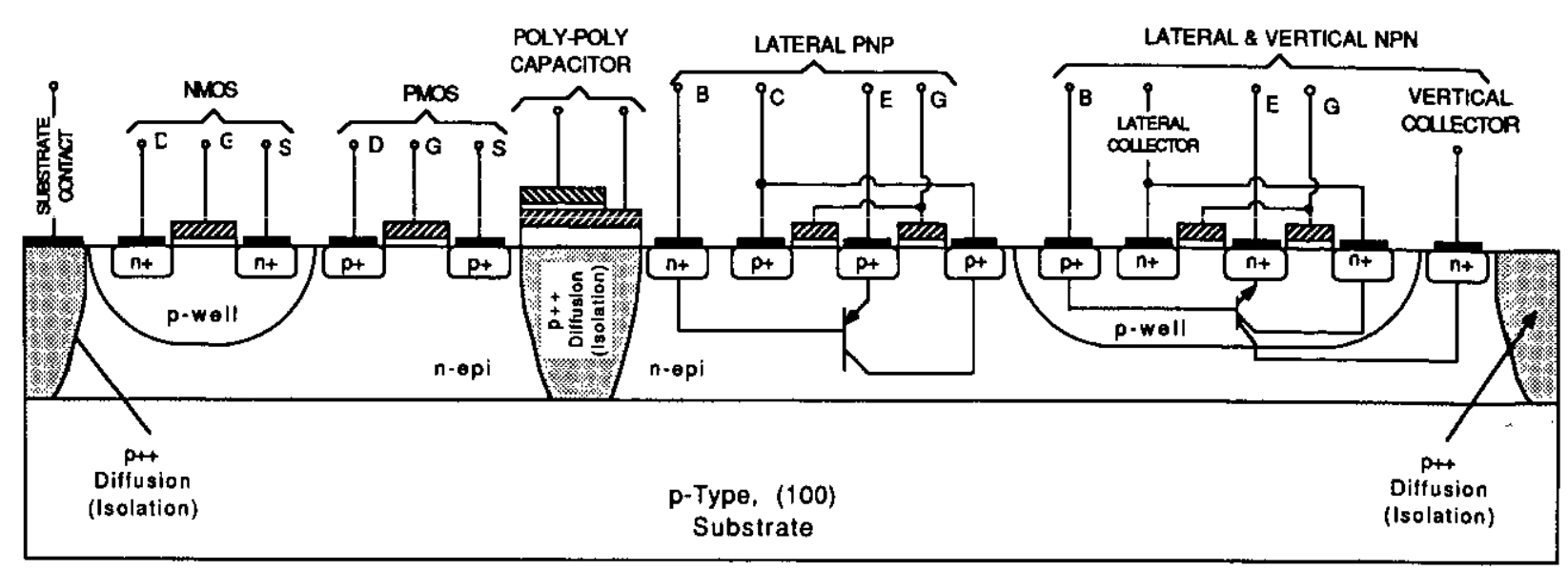

Figure 4. Cross-sectional view of a cMOs-bipolar micromachining compatible fabrication technology for use in smart sensors [27].

try is integrated on a $\mathrm{n}$ epi layer that is grown over a $\mathrm{p}$ substrate. This layering combination not only permits the fabrication of the cmos circuitry, but also allows a number of micromachining steps to be performed to fabricate a variety of microstructures. Deep boron diffusion from the front side of the wafer allows the use of boron etch-stop techniques to be used for forming beams and diaphragms, while electrochemical etch stops between the $n$ epi layer and the $p$ substrate allow the fabrication of a variety of other microstructures, including diaphragms, beams, etc. Ishihara et al have used this technique to fabricate CMOS circuitry together with a piezoresistive pressure sensor by using electrochemical pn junction etch stops [5]. Although this sensor operates solely using the cMOS circuitry, it is possible to incorporate the parasitic bipolar devices to implement a number of different circuit functions requiring BJTs.

Our group at the University of Michigan has developed a standard $3 \mu \mathrm{m}$ bulk p well cMos process together with deep boron diffusion and boron etch stop to fabricate multichannel active recording electrodes for biomedical applications [8]. Figure 5 shows the fabrication sequence for an active 32-channel electronically configurable multielectrode recording array with on-chip CMOs signal processing circuitry, while figure 6 shows a photomicrograph of the fabricated chip. The process utilizes a total of 10 masking steps and results in highperformance $\overline{\mathrm{CM}} \overline{\mathrm{O}} \overline{\mathrm{S}}$ devices and circuitry. In this process, the cMOs circuitry is integrated in the lightly-doped $n$ epi layer grown over a p substrate. A deep boron diffusion is performed which diffuses through the $n$ epi layer and connects to the underlying $\mathrm{p}$ substrate, thus creating isolated $n$ epi regions that will house the circuitry. These $\mathrm{p}+$-diffused regions will protect the sensor from lateral etching during the EDP etching process, while the etch from the back side is timed to prevent the removal of the p substrate. In addition, the p +-diffused regions can be ùsed to providue jưnction isolation for the cmos portion of the circuitry and to create isolated $n$ epi regions that form the collector of high-performance vertical npn BJTs, as shown in figure 4 . We have obtained vertical npn bipolar devices with a current gain in the range 100-150 using the above process [27]. In addition to the vertical transistors, one can utilize lateral npn and pnp bipolar transistors that are naturally formed through the cmos process [28]. Both parasitic vertical and lateral bipolar devices have been utilized in integrated sensors and have been used to implement such functions as temperature sensors [20].

This CMOs technology utilizes two polysilicon layers in order to implement on-chip integrated capacitors which are often required in many switched capacitor and analog circuits. In addition, it allows the use of refractory interconnections instead of the conventional aluminum, when desired. Refractory interconnects are desirable in many sensor/actuator applications since they can be further insulated on top with low-pressure chemical vapor deposited (LPCVD) thin films thus allowing the entire sensor/circuit structure to be isolated from destruuctîive and ốten corrosive external environments. In effect, LPCVD nitride and oxide films provide a long-term non-hermetic package for the circuit while still allowing the sensor to interface with the external world for parameter measurement.

Compatible integration of the sensor and the circuitry, is perhaps one of the main concerns when fabricating sensors with on-chip circuitry. The combined sensor/circuit process should be such that the circuit process is minimally interrupted by the sensor process, while still allowing precise micromachining of the silicon substrate. The ideal merging of the sensor and the circuit processing is best achieved when the sensor process is performed at the beginning or the end of the circuit process. In the sensor-CMOs-bipolar process described above, etch stops are formed at the beginning of the process in order to avoid exposure of the circuit to high thermal budgets required for the deep boron diffusion, followed by a nearly unaltered circuit process, in this case cMos. The final silicon etch completes the process. With such a sequence, minimal change is required in the normal circuit recipe. In the case of a deep boron sensor diffusion (for a diaphragm or other microstructures), the $p$ well drive in can be performed simultaneously with the boron etch-stop diffusion. For sensors fabricated using 


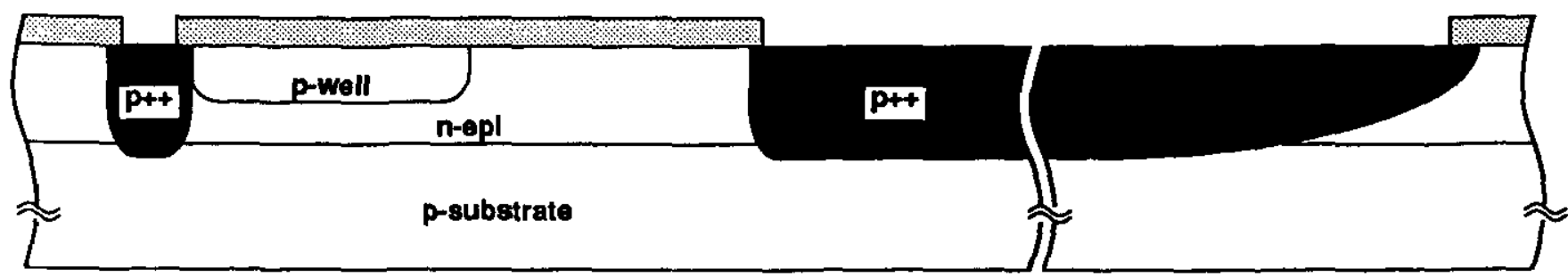

(a)

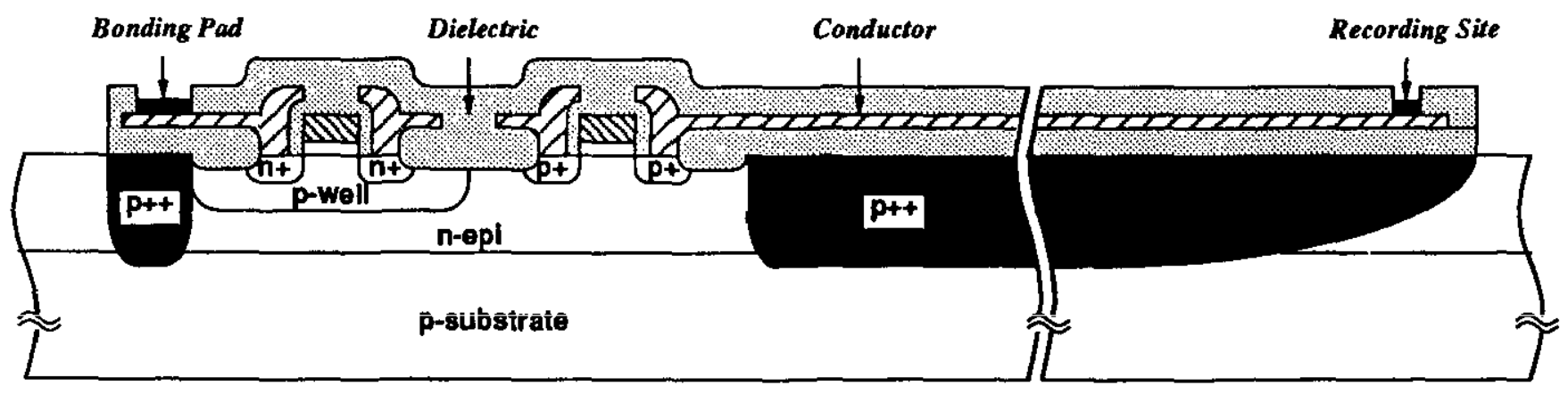

(b)

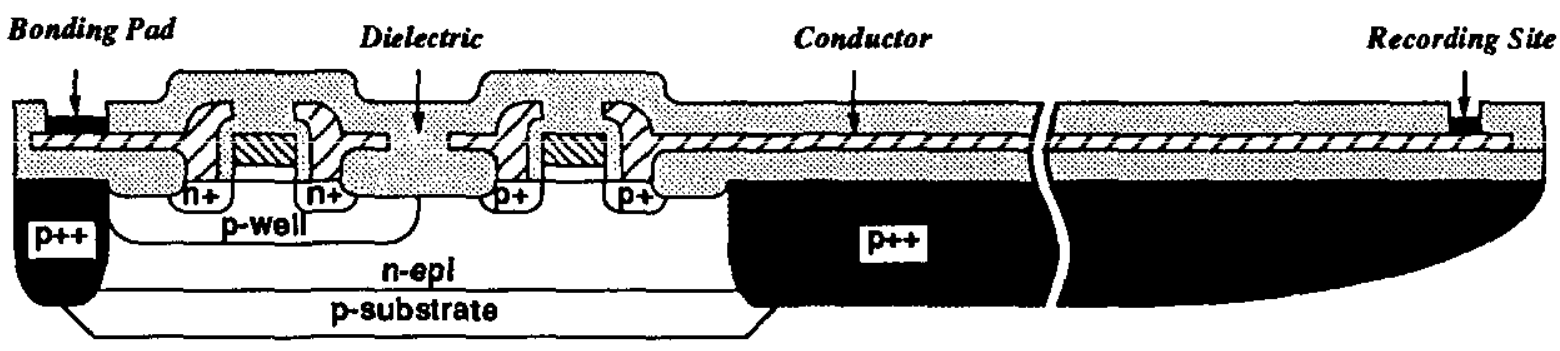

(c)

Figure 5. Fabrication sequence for an active multichannel recording electrode with on-chip cMOs circuitry (a) Selectively diffuse substrate to define probe shape; $(b)$ process circuitry and pattern electrodes; $(c)$ etch substrate and separate probes. (Reprinted courtesy of IEEE [8].)

surface micromachining technologies, it is also possible to incorporate electronics with the sensor. A number of groups worldwide have demonstrated sensor-circuit integration using a standard cMOs process $[29,30]$. Thus in most cases, while merging the transducer and the circuit processes requires careful study and possibly altering individual process steps, it can usually be accomplished.

\section{Packaging}

The package for an integrated sensor should satisfy several requirements that are sometimes in conflict. First, it must provide the sensor with access to the parameter of interest, while protecting the interface electronics from the potentially hostile environment. It must also provide elegctrical access to the interface circuits for data, control and power. For biomedical applications, the package should satisfy the added requirement of not adversely affecting the biological tissue with which it is in contact. In addition to these requirements, the package must be small, cheap and stable over time. Indeed, the package cost for many sensors can easily become a major part of the overall cost and can degrade the performance characteristics of the sensor. Therefore, careful attention to the design of the package should be paid at an early stage of the sensor design process to alleviate some of these problems.

Packaging techniques for smart sensors can be divided into two categories. Many present sensors fall into the first category where a standard outline IC package has been modified. DIP packages and To cans have been used 


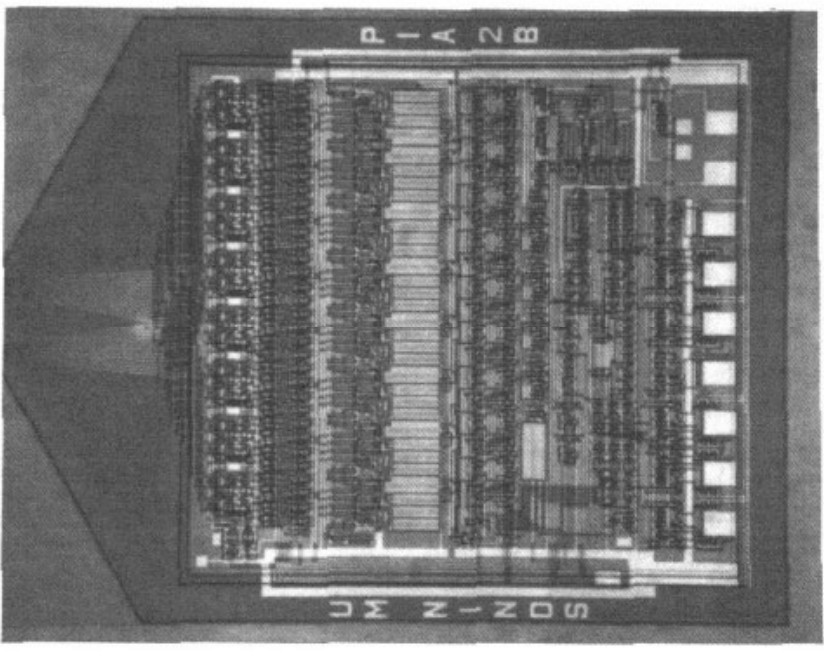

Figure 6. Photograph of the back end of a secondgeneration 32-channel active recording microprobe. (Reprinted courtesy of IEEE [8].)

for some commercial sensors with minor modifications [16], while some custom packages have been designed for other sensors, including pressure sensors, using injection molding $[31,32]$. It is believed that these packaging techniques will be utilized to a large extent in the future to package smart sensors, especially if selective encapsulation of sensor electronics is not required. Accelerometers are perhaps one of the few sensors that benefit most from the already available packages and techniques available for integrated circuits.

For many other sensors, however, the use of conventional IC packages is not desirable, and different packaging schemes have to be developed. As mentioned above, for some sensors such as accelerometers, magnetic-field sensors, visible imagers and some temperature sensors, the device can be encapsulated by a hermetic metal or metal-glass can. However, for other sensors, such as pressure, gas and chemical sensors, the device must be in direct contact with the environment. The refore, selective encapsulation is required rather than overall protection from the environment. There is one environmental parameter against which one cannot protect in most packages, namely temperature. This is perhaps the most important parameter challenging future development of smart sensors. If the sensor has to operate in a bightemperature environment, the electronic circuitry should also be able to withstand the high-temperature environment. Most notable of these applications is under-thehood automotive sensors. A large amount of effort is now being expended by the electronics industry to extend the high-temperature operation range of IC circuits using a variety of technologies including improved layout and fabtication techniques [33], and silicon-on-insulator circuit approaches [34]. This trend is certainly of great importance to smart sensors. The package for a smart sensor should be capable of encapsulating the circuitry from other environmental factors such as moisture. In some implantable biomedical applications, the sensor package should satisfy the added requirement of being very small, approaching the size of the silicon chip itself. For many of these new applications, new packaging techniques that utilize technologies such as wafer-towafer hermetic bonds and polymer or inorganic chip coatings are being utilized.

One technique for encapsulating smart sensors at the wafer level utilizes hermetic sealing of two or more chips (wafers) to provide a hermetic seal that can protect the electronics. Silicon-glass electrostatic bonding [35], silicon-silicon eutectic bonding [16], or silicon-silicon electrostatic bonding using an intermediate sputtered glass layer $[36,37]$ have all been used and illustrated to satisfy the needs of their particular application areas. The advantage of many of these packaging techniques is that they can be performed at the wafer level and can be performed selectively over the electronics. Recent attempts have used custom designed glass packages for hermetic packaging of the electronics while providing sealed feedthroughs for access to the electronics, and allow access between the sensor and the environment of interest [38]. Another packaging technology that has received attention over the past few years is that of nonhermetic sealing of ICs using organic and inorganic thin films. This is of particular interest to the military, and major progress has been made in this area over a very short period. A variety of materials including deposited inorganic thin films such as silicon nitride and organic thin films such as parylene and spin-on glass have been used in various applications and early results indicate that the electronics can be adequately protected against moisture and ionic contamination for extended periods of time. This type of packaging may be ideal for sensor applications since the package is small, can be formed at the wafer level and can be performed selectively over the electronics. These films are also very important in many smart implantable biomedical sensors where package size should be kept as small as possible [39].

Packaging of solid-state sensors in general, and packaging of smart sensors in particular, will remain one of the most challenging problems facing the sensor community. However, it should be noted that by integrating on-chip circuitry it is possible to amplify and buffer the recorded signals before transmission to the outside world and relieve the package from having to protect otherwise weak signals. This is even more important for long-term reliability of the sensor. For multisensor devices, multiplexing of several data channels onto a single data line not only relieves packaging and encapsulation but also improves the lifetime and yield of the final product by minimizing the number of leads and bonds, which are potential points of failure. Therefore, the sensor designer should weigh the advantage of improved performance and reduced lead count when incorporating the circuitry into the sensor module against the disadvantage of having to protect this circuitry against harsh environments encountered by the sensor. It is believed that for many applications the advantages of incorporating signal processing circuitry at the sensor site will outweigh the disadvantages. 


\section{Examples}

Although the development of truly smart sensors has been a relatively new trend, and prototype smart sensors remain to be demonstrated, integration of on-chip circuitry and solid-state sensors has been illustrated by many researchers for a number of years. One of the first attempts at fabricating an integrated sensor was by Borky and Wise [40]: a piezoresistive pressure sensor based on a resistive bridge and a bipolar amplifier were integrated on a silicon diaphragm formed using isotropic etching. This work in pressure sensors was followed by the development of a series of pressure sensors incorporating varying amounts of on-chip circuitry and finally paved the way for the development of improved integrated pressure sensors that were commercially produced [41]. Most of these integrated sensors utilized only a minimal amount of circuitry, typically in the form of a single amplifier, in order to keep cost down and maintain high yield. However, as sensor and circuit technologies matured and as sensor designers gained more experience with circuit and sensor design and fabrication, the level of sophistication of on-chip circuitry on integrated sensors increased. The following three examples represent integrated sensors developed at the University of Michigan and illustrate the implementation of some of the features of smart sensors in actual devices.

\subsection{Active multielectrode intracortical recording probes}

One of the first integrated sensors that incorporated a substantial amount of signal processing circuitry on the sensor chip was an active multichannel recording microprobe developed for recording extracellular activity of brain cells [42]. The use of microelectrodes to record extracellular biopotentials generated electrochemically within individual neurons has been one of the principal techniques for studying the central nervous system at the cellular level. Long-term recording of electrical discharges from several neurons simultaneously will help us gain more understanding of the neural systems at the

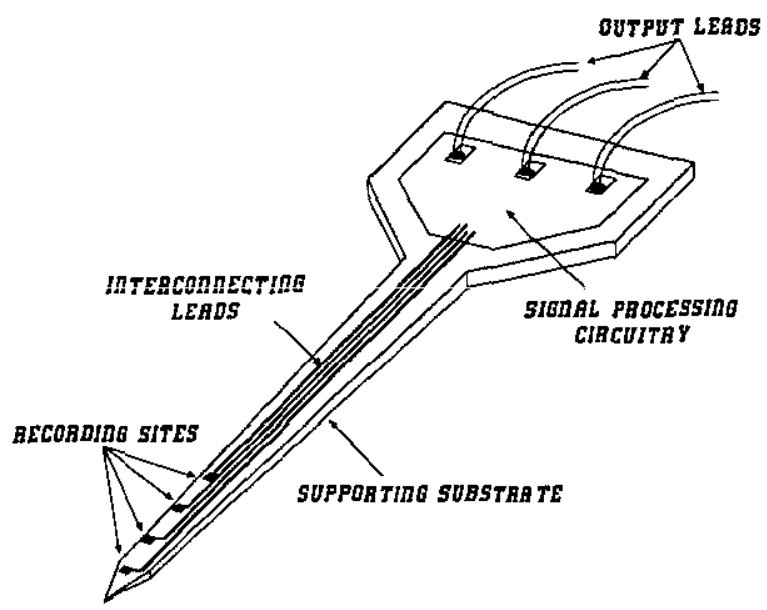

Figure 7. Structure of a silicon-based multichannel recording microprobe.

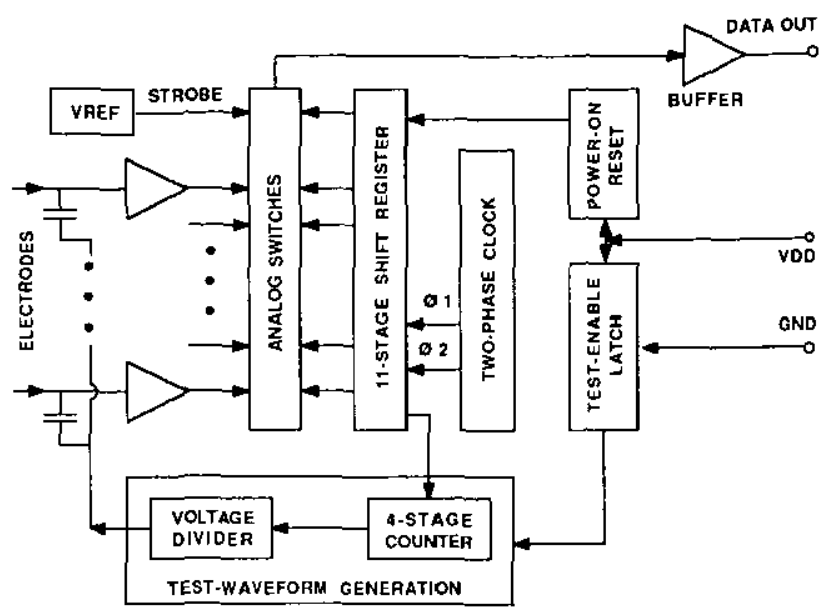

Figure 8. Block diagram of the on-chip circuitry for the multichannel recording microprobe.

circuit level. Therefore, an implantable multielectrode recording microprobe that can record neural activity over an extended period of time is required. Figure 7 shows the structure of a micromachined silicon microprobe developed for this application by our group. The micromachined silicon substrate supports an array of recording sites that interface with individual neurons and record their electrical discharges. The recorded signals are fed to a bank of per-channel amplifiers that amplify the recorded signals and filter out the high frequency noise. Figure 8 shows the block diagram of the on-chip circuitry that is integrated on the rear of the probe substrate. The silicon substrate is formed using deep boron diffusion and boron etch-stop techniques. Boron doping is also used around the perimeter of the rear of the probe for dimensional control of the probe, while a silicon well of normal resistivity is retained for circuit fabrication.

The on-chip circuitry consists of ten per-channel preamplifiers followed by an analog multiplexer and a broad-band output filter to drive the external data line. In order to allow the external regeneration of the on-chip clock, a synchronization pulse is inserted once each frame as an eleventh channel. The external electronics strips off these pulses, regenerates the sample clock, and demultiplexes the neural signals for external recording and processing equipment. The on-chip electronics is also capable of self-testing the electrode impedance levels on demand. When testing is desired, the power supply is pulsed from its normal value of $5 \mathrm{~V}$ to $8 \mathrm{~V}$, enabling the test-waveform generation circuitry. This circuit generates a $50 \mathrm{mV}, 1 \mathrm{kHz}$ signal that is capacitively coupled to all the recording electrodes. The resulting induced electrode voltages are amplified and multiplexed out in the normal way, providing an indication of the electrode impedance levels and of the functionality of the on-chip electronics. After testing is performed the circuitry can be returned to its normal mode by turning the power supply off and then back on again.

The design for the on-chip circuitry requires only three output leads for power, ground and data while 
maintaining self-test capability. The fabrication technology chosen for this first generation active probe was a $6 \mu \mathrm{m}$, single-metal, triple-implanted, LOCOS ED NMOS process. Figure 9 shows a scanning electron microscope view of a fabricated ten-electrode array with associated onchip circuitry. The probe is $15 \mu \mathrm{m}$ thick and $4.7 \mathrm{~mm}$ long overall, with a $3.2 \mathrm{~mm}$ long shank that tapers from a width of $160 \mu \mathrm{m}$ at the bottom to less than $20 \mu \mathrm{m}$ near the tip.
A second-generation 32-channel microprobe has also been developed [8] that extends the functionality of the on-chip circuitry and scales the overall dimension of the probe shank to reduce damage to the tissue. This secondgeneration design utilizes a $3 \mu \mathrm{m}$ p well CMos process to realize the on-chip circuitry that maintains the three-lead system designed for the first-generation microprobe, while allowing for the probe to be programmed externally. Figure 10 shows the block diagram of the circuitry

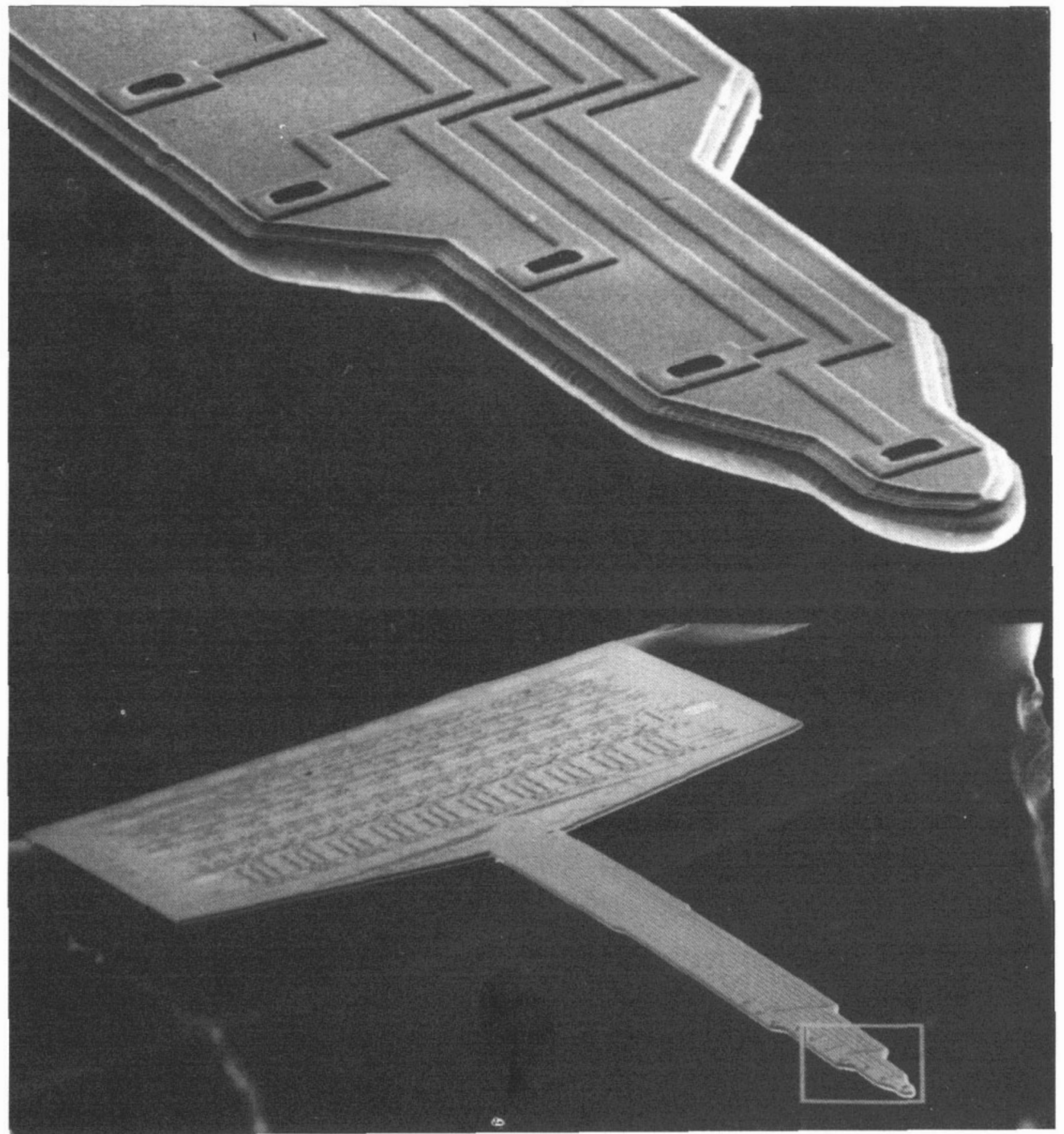

Figure 9. SEM views of a ten-electrode silicon-based recording microprobe with on-chip circuitry. 


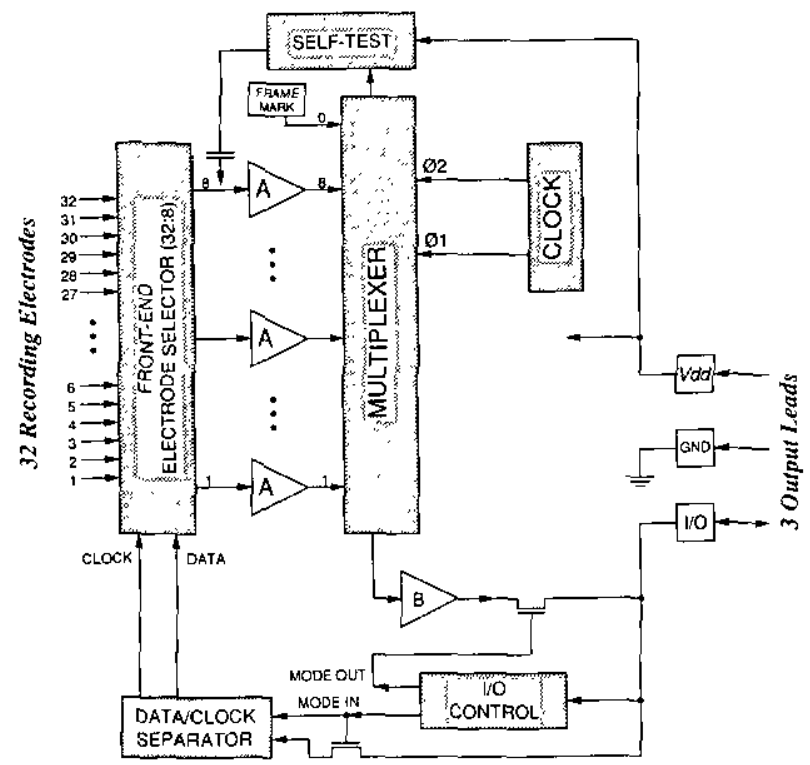

Figure 10. Block diagram of the on-chip circuitry for the second-generation, programmable, 32-channe! recording microprobe. (Reprinted courtesy of IEEE [8].)

and figure 6 shows a photomicrograph of a fabricated probe. An input channel selector is used to select eight active recording sites from among 32 sites located on the probe. This approach allows the channels selected for processing to be optimized for maximum electrode-cell coupling and effectively implements electronic site positioning. The eight selected sites feed their recorded data to per-channel amplifiers for amplification whose outputs are subsequently multiplexed onto a single data line that is transmitted to the outside world. The amplifiers are designed with low-pass filters implemented using a new diode-capacitor approach. These filters are required to filter out the DC input drift generated by the recording site-tissue interface and the amplifier offset voltage. This will permit the amplifiers to be designed for maximum $\mathrm{AC}$ gain while avoiding saturation due to these DC offsets. Channel selection is obtained over the same lead normally used for multiplexed data output from the probe. A $5 \mathrm{~V}$ pulse applied externally to this lead selects its mode. In the output mode, the eight signal samples and frame marker are time-multiplexed to the external circuitry, while in the input mode binary input data and clock signals are superimposed on the data lead. On-chip circuitry separates these signals and clocks the input channel selector to enter the desired selection mode. Onchip self-test capability is also maintained in this second generation probe [8].

These two integrated sensors represent an important step forward in the implementation of smart sensors. They perform some of the most important functions required for many smart sensors. Signal amplification, filtering, multiplexing, self-testing and calibration, and bidirectional communication with a higher level controller are among their most important features. Perhaps as important as the circuit functions is the integration of the sensor and circuit process into a single high-yield process. Boron etch-stop and CMOS/NMOs fabrication technologies are combined to implement a device that offers the best of silicon micromachining and IC fabrication technologies.

\subsection{Multi-element thermally based sensors}

Thermally based sensors are widely used in a variety of applications to measure gas flow, temperature, infrared radiation and a number of other parameters $[20,43]$. Most of these sensors utilize silicon micromachining techniques to create a thermally isolated area where a specific sensor is located and is subjected to the environment of interest. One such sensor developed by researchers at the University of Michigan is a multielement monolithic mass flowmeter with on-chip signal processing electronics [20]. Figure 11 shows a crosssectional view and plan of this sensor. The transducing structures are fabricated on dielectric diaphrams that provide thermal isolation from the support rims. Four sensing structures are implemented to measure gas flow, flow direction and gas type. Two sensors to measure flow velocity use a thin gold-chromium film as a temperature detector and polysilicon as a heater. Flow direction is measured using a third window, which contains two orthogonal pairs of polysilicon-gold thermopile detectors together with a polysilicon heater. The fourth window contains a conductivity cell for the measurement
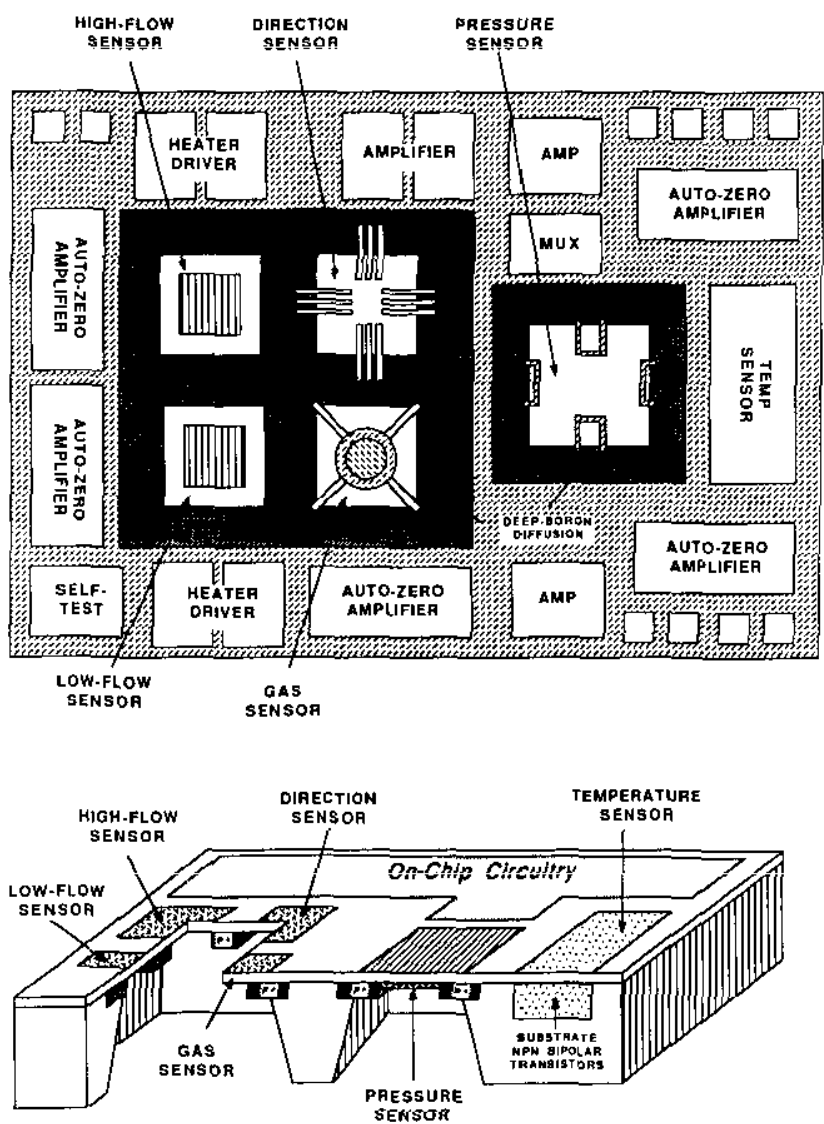

Figure 11. Cross-sectional view and plan of a multielement thermally-based mass flowmeter. (Reprinted courtesy of IEEE [20].) 
of gas type [44]. A piezoresistive polysilicon pressure sensor is located in a separate well for the measurement of ambient pressure.

The outputs of these sensors are received by the onchip circuitry which is fabricated on the unetched bulk portion of the silicon chip. Figure 12 shows the block diagram for the on-chip circuitry. Switched-capacitor offset-zeroing amplifiers are used to amplify sensed signals and to filter out DC offset levels. The thermally-based sensors (for flow velocity, flow direction and gas type) operate in constant temperature mode, with temperatures set by on-chip feedback loops and heater drivers. After amplification, the sensor outputs are multiplexed out and transmitted to the outside world through an output buffer. Self-testing circuitry is also implemented on-chip to measure changes in the electrothermal characteristics of the diaphragm windows in-situ. An on-chip temperature sensor implemented using CMos and parasitic bipolar devices available in the bulk cMos process is used to measure the substrate temperature and provide a feedback signal for heater control. This circuitry requires a total of ten output leads and dissipates $120 \mathrm{~mW}$ of power from $\pm 5 \mathrm{~V}$ supplies and measures $3.5 \mathrm{~mm} \times 5 \mathrm{~mm}$ in a $3 \mu \mathrm{m}$ bulk p-well CMOs technology. The windows are defined using deep boron diffusion and the diaphragms are formed by anisotropic etching from the back side of the wafer. Figure 13 shows a photomicrograph of the finished sensor showing the five sensors and the on-chip circuitry. One of the major functions of this on-chip circuitry has been the reduction of output leads as without it the mere number of different sensors required for true mass flow measurement would have overburdened the final packaging and assembly process. The same technology described above is also used to fabricate a monolithic RMS-DC converter [45].

These devices clearly illustrate the capabilities of onchip circuitry in providing amplification, filtering and multiplexing, as well as feedback control for sensors and actuators. In addition, the on-chip circuitry is an integral part of this particular sensor and helps provide such functions as temperature sensing and heater control.

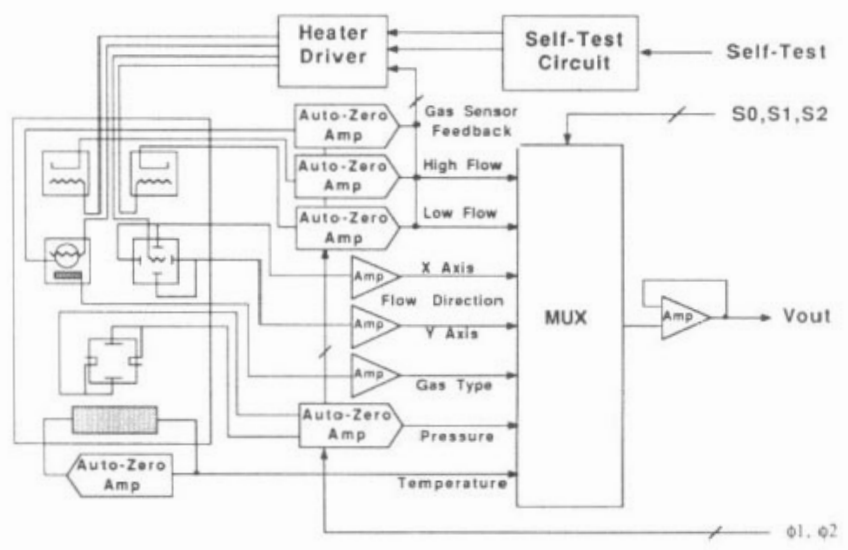

Figure 12. Block diagram of the on-chip circuitry for a thermally-based mass flowmeter. (Reprinted courtesy of IEEE [20].)

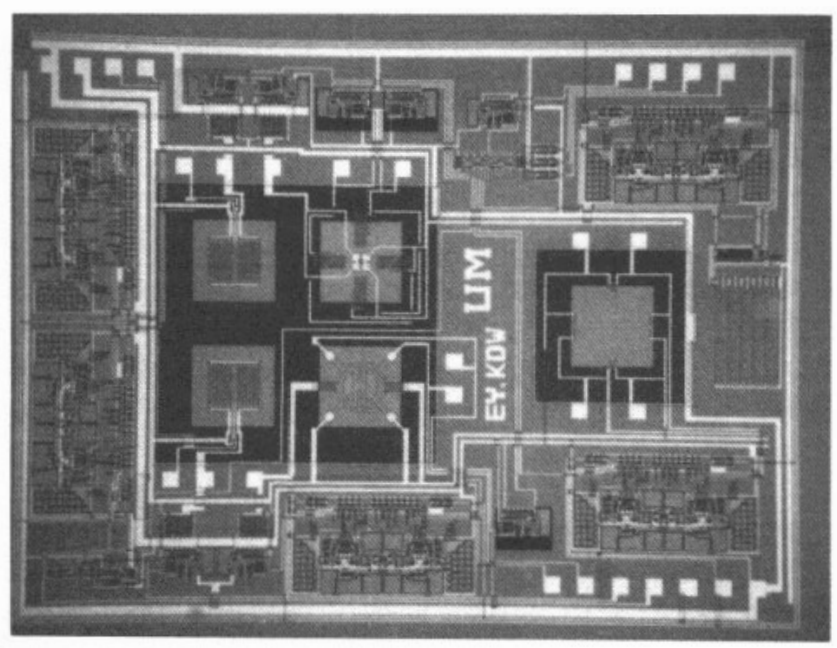

Figure 13. Photomicrograph of a multi-element mass flowmeter fabricated using bulk silicon micromachining and bulk $p$-well cMos technologies. (Reprinted courtesy of IEEE [20].)

Indeed, it is believed that on-chip circuitry will be increasingly used to test, diagnose and calibrate microsensors and microactuators to improve accuracy, reliability and long-term stability. The above integrated sensors have maintained an analog interface with the outside world, and require a standardized interface with an external processor which is custom-designed for their particular applications. It is extremely desirable to standardize the external interface through a digital bus thus allowing the host controller to communicate with the sensor, and implement more sophisticated functions that are desirable in fifth-generation sensors. Efforts are currently underway to develop such sensors.

\subsection{VLSI smart sensors}

An important requirement for many future sensing systems is true compatibility with microprocessor driven instrumentation/control systems. The sensors utilized in these systems should be capable of performing many computationally intensive functions at the sensor site and should be able to communicate with the controlling processor through a bidirectional bus, thus making them behave more like a system periphery than a passive component whose condition is generally unknown to the controller. Therefore, in addition to the standard signal processing functions such as amplification and multiplexing, these sensing systems should be capable of converting the sensed signal into a digital form and should be capable of manipulating the data at the command of the controller before transmission to the controller over the digital bus. The question of whether the required circuitry should be integrated monolithically on the sensor chip or hybrid connected to the sensor chip in a multi-chip module will be determined by such issues as cost and performance requirements for the particular application. In either case, from the system designer's standpoint this is unimportant as the standard 


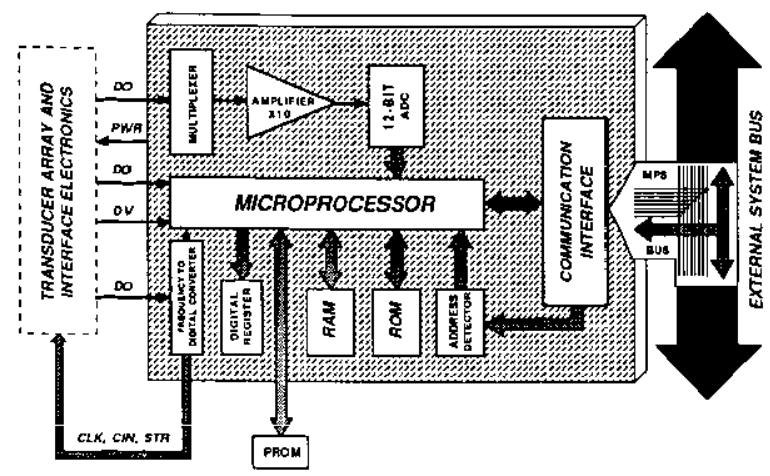

Figure 14. Block diagram of a smart sensor interface chip. (Reprinted courtesy of IEEE [46].)

interface offered by the sensor to the system is the primary feature. One of the best efforts aimed at the development of appropriate architectures and interfaces for smart sensing systems is described in [21]. The following will provide a summary of this work.

The overall architecture for a VLSI smart sensor was shown in figure 3 , and the block diagram of a monolithic smart sensor interface chip under development at Michigan $[21,46]$ is shown in figure 14 . The first-generation design of this chip accepts voltage-amplitude inputs from a front-end transducer chip, which are amplified and digitized under the control of a custom-designed microprocessor. The chip also accepts digital (event) inputs as well as pulse-rate (FM) inputs. Digitized sensor data is stored in on-chip RAM. An off-chip PROM stores the program code for the processor, personality information regarding the variables measured, the compensation techniques to be used with the data and the compensation coefficients themselves. The interface between the processor chip and the PROM is implemented in parallel to allow the use of a commercial PROM, in this case a 2716. The communication interface is configured for the Michigan parallel standard (MPS) developed at the University of Michigan [21]. The processor here employs a 12-bit internal buss, consistent with handling sensor data at the 12-bit level. Table 1 summarizes the characteristics of this interface chip in a $3 \mu \mathrm{m}$ single-metal double-poly смоs technology. The microprocessor and communication interface are custom designed, while the amplifier and $A D C$ are realized with the aid of a silicon compiler. Substantial compaction of this circuit is thought to be possible as circuits are further optimized and as finer feature sizes are used for the circuitry. The amplifier is designed for an open loop gain of $87 \mathrm{~dB}$ and a closed loop gain of $20 \mathrm{~dB}$. The switched-capacitor analog-digital converter has a simulated conversion time of $13.5 \mu \mathrm{s}$ at 12 bits and a clock rate of $8 \mathrm{MHz}$. A discrete version of this interface chip has been implemented, is fully functional and is used to obtain a variety of sensor data through a digital bus and a personal computer.

Because of the rather large area and complexity of this first generation design, N Najafi et al [21] have designed a second-generation interface chip whose characteristics are also summarized in table 1 . The main difference between these two designs is that the secondgeneration chip accepts either digital data or frequency data only (it is assumed that the transducer chip produces a digital data stream using an on-chip ADC before interfacing with the smart sensor chip). Therefore, an ADC is not required, which makes the interface chip all digital. The technology used here is a $1.25 \mu \mathrm{m}$ double-metal single-poly CMOS, and the chip runs from a single $5 \mathrm{~V}$ supply, occupies $11.7 \mathrm{~mm}^{2}$ and requires only 12 pads. This second-generation design utilizes a serial interface, thus reducing the number of output leads drastically [21].

Although these sensor interface chips represent the most sophisticated and versatile smart sensors developed to date, other research groupss are developing señsor interface chips that attempt to achieve similar functions $[47,48]$. Many of these designs and sensing systems illustrate the need and power of smart sensors and will eventually pave the way for the development of integrated sensing systems compatible with computerbased instrumentation and control systems.

\section{Conclusion}

We have observed tremendous growth and progress in solid-state sensor technology during the past two decades. This progress is brought about principally by the

Table 1. Characteristics of two sensor interface chips for use in smart sensors [46].

\begin{tabular}{lll}
\hline Parameter & First smart sensor interface & Second smart sensor interface \\
\hline Technology & $3 \mu \mathrm{m}, 1$-metal, 2-poly, cMOS & $1.25 \mu \mathrm{m}, 2$-metal, 1-poly, cMOS \\
Supply voltages & $+5 \mathrm{VHz}$ & $+5 \mathrm{~V}$ \\
$\mu$ proc clock frequency & 63 & $8 \mathrm{MHz}$ \\
No. of pads & $8-\mathrm{bit}$ & 12 \\
FDC conversion accuracy & $14 \mu \mathrm{s} \mathrm{@12-bits}$ & $12-\mathrm{bit}$ \\
ADC conversion time & $20 \mathrm{~mm}^{2}$ & - \\
ADC area & $87 \mathrm{~mm}^{2}$ & $11.7 \mathrm{~mm}^{2}$ \\
Active chip area & MPS (parallel) & MSs (serial) \\
Bus interface & Parallel (2716) & Serial (4-line) \\
PROM interface & $8-$ line standard & $8-$ line standard \\
Input interface & Analog-level, binary word, frequency & Binary word, frequency \\
Input data types & & \\
\hline
\end{tabular}


development of silicon micromachining techniques and the utilization of the wide array of technologies already in place for the fabrication of integrated circuit chips. These sensors are potentially cheaper, offer higher performance and reliability, and are much smaller in size than their discrete counterparts. They have been employed in applications including transportation and health care and in large part have fulfilled their promise. They remain to be widely applied in all areas of instrumentation and control due to a number of interrelated and complex factors including the initial cost of research and development, the effort and cost required to upgrade existing systems that is necessary to accommodate the new sensor, and the training and effort required to train engineers and system designers to enable them to take full advantage of the new device. As a result, many solidstate sensors have often been used as direct replacements for their discrete predecessors and in the process have not realized their full potential. Indeed, many of the most successful solid-state sensors have resulted from a complete redesign and overhaul of the system environment in which they operate. It is in this context that the author believes smart integrated sensors will see continuing growth and development.

Smart sensors take full advantage of the worlds of solid-state sensors and integrated circuits by combining the best features these have to offer. Sensor signals can be amplified and properly processed, are multiplexed and are buffered ready to be received by microprocessorbased systems. Computational and control logic operate on these signals and offer a standard data stream to the user thus making the entire sensing module behave like a system periphery rather than a passive component. The system designer can now operate the sensing module as one that can be manipulated remotely and programmed for self-testing, calibration, and for performing specialized tasks. This added flexibility and versatility will, in turn, reduce system overheads in terms of cost, configuration and software requirements, and will immensely crihance overall stability, reliability and performance.

Many obstacles still remain to be overcome before smart sensors become commonplace. First, and perhaps most important, is the further development of truly compatible sensor-circuit fabrication processes so that the overall yield can be maintained at a high level and the total cost can be kept low. Second, the fabrication cost of smart sensors should be reduced. This will require the fabrication of solid-state sensors using specialized equipment, and the fabrication of ICS using fabrication foundries in order to avoid the large capital investment required for integrated circuit fabrication. Two markets will be more likely to see the emergence of smart sensors: very high performance sensor applications where the unit cost is not as important as the level of performance achieved by the sensor, and high volume markets where both high-performance and low cost are desired, such as medical and consumer electronics which have the potential for very large volumes. The very high volume automotive market requires that the unit cost of the sensor be kept low. These high-volume markets are better able to absorb the initial development and capital investment; although they are also more sensitive to unit cost. Third, computer-aided design tools need to be developed for sensor design and simulation. This is a very important aspect of the development of a smart sensor since one can check the functionality of both the sensor and the circuit before committing to fabrication. A number of programs around the world are developing such CAD tools for sensor/actuator design, including our program at the University of Michigan where a fully integrated CAD system for microelectromechanical system design is being developed [49]. Fourth, standardization of sensing systems and their various modules are badly needed and should be pursued at all levels. The question of standard architectures and interfaces are being addressed by a number of groups [21]. Standard circuit modules and interfaces need to be developed in order to avoid design duplications and reduce the overhead when a new sensor is to be developed. This will inevitably require closer cooperation between sensor designers, circuit designers, system designers and process engineers. It is interesting that such cooperation and interaction was also required for the development of many VLSI circuits and systems.

The author believes that the promise of solid-state sensors will be fully realized when they are integrated into closed loop instrumentation systems incorporating both microsensors and microactuators. Many of the advantages and advanced functionality of solid-state sensors can only be realized by considering the overall system. Development of these integrated systems will be possible through the development of 'smart sensors'.

\section{Acknowledgments}

The review presented in this paper has been based on the collective efforts of a large number of individuals all around the world who have over the years worked on the development of solid-state sensors. In particular, a major effort in the research and development of solid-state integrated sensors has been made here at the University of Michigan under the direction of Professor Kensall D Wise. A great many individuals have been involved in the completion of various projects reported here, including Dr J Ji who has played a major role in the development of active multielectrode recording probes and sensorcircuit fabrication technologies, Dr E Yoon who has developed the thermally-based sensors, Mr N Najafi who has developed the visi smart sensor interface and standards and $\mathrm{Mr}$ Y Gianchandani who has been responsible for the development of the bipolar cMosmicromachining process. Special thanks go to our sponsors who have provided us with the encouragement and means to carry out our research programs. The author gratefully acknowledges Dr T Hambrecht and Dr W J Heetderks of the National Institutes of Health for their support of our biomedical sensor projects, $\mathrm{Dr} G$ Hazelrigg of the National Science Foundation and many individuals at the Semiconductor Research Corporation. 


\section{References}

[1] Brignell J E 1989 Smart sensors Sensors, A Comprehensive Survey ed W Gopel, J Hesse and J N Zemel vol 1 (New York: VCH)

[2] Giachino J M 1986 Smart sensors Sensor and Actuators $10239-48$

[3] Tredwell T J High-density solid-state image IEEE Int. Conf. on Solid-State Sensors and Actuators (Philadelphia, 1985) Technical digest pp 424-9

[4] Sugiyama S, Takigawa M and Igarashi I 1983 Integrated piezoresistive pressure sensor with both voltage and frequency output Sensors and Actuators $4113-20$

[5] Ishihara T, Suzuki K, Suwazono S, Hirata M and Tanigawa $\mathrm{H}$ CMOS integrated silicon pressure sensor IEEE J. Solid-State Circuits SC-22 151-6

[6] Gray P R and Meyer R G 1982 Mos operational amplifier design-a tutorial overview IEEE J. SolidState Circuits SC-17 969-83

[7] Hsieh K C, Gray P R, Senderowicz D and Messerschmitt D G 1981 A low-noise chopperstabilized differential switched-capacitor filtering technique IEEE J. Solid-State Circuits SC-16 70815

[8] Ji J and Wise K D An implantable cmos analog signal processor for multiplexed microelectrode recording arrays IEEE Solid-State Sensor and Actuator Workshop (Hilton-Head, SC, 1990) Technical Digest pp 107-10

[9] Lyman J 1987 Smart sensors will help cut wiring that clogs systems Electronics 73-5

[10] Brignell J E Sensors in distributed instrumentation systems 1986 Sensors and Actuators $10249-61$

[11] Degrauwe M G R, Nys O, Dijkstra E, Rijmenants J, Bitz S, Goffart B L A G, Vittoz E A, Cserveny S, Meixenberger $C$, van der Stappen $G$ and Oguey $H$ J 1987 IDAC: an interactive design tool for analog CMOS circuits IEEE J. Solid-State Circuits SC-22 1106-15

[12] McCreary J L and Gray P R 1975 ALL-Mos chargeredistribution analog-digital conversion techniques-Part I IEEE J. Solid-State Circuits SC$10371-9$

[13] Suarez R E, Gray P R and Hodges D A 1975 ALL-mos charge-redistribution analog-digital conversion conversion techniques-part II IEEE J. Solid-State Circuits SC-10 379-85

[14] Lee H S and Hodges D A 1983 Self-calibration technique for $\mathrm{A} / \mathrm{D}$ converters IEEE Trans. on Circuits and Systems CAS-30 (New York: IEEE) pp $188-90$

[15] Lee H S and Hodges D A 1985 Accuracy considerations in self-calibrating A/D converters IEEE Trans. on Circuits and Systems CAS-32 (New York: IEEE) pp 590-7

[16] Allen H V, Terry S C and de Bruin D W Self-testable accelerometer systems IEEE Micro Electro Mechanical Systems Workshop (Salt Lake City, Utah, 1989) Technical digest pp 113-5

[17] Cho S T, Najafi K, Wise K D Scaling and dielectric stress compensation of ultrasensitive boron-doped silicon microstructures IEEE Micro Electro Mechanical Systems Workshop (Napa, CA, 1990) Technical digest pp 50-5

[18] Crary S B, Baer W G, Cowles J C and Wise K D 1990 Digital compensation of high-performance silicon pressure transducers Sensors and Actuators A21-3 $70-2$

[19] Regtien P P L and Trimp P J 1990 Dynamic calibration of sensors using EEPROMS Sensors and
Actuators A21-3 615-8

[20] Yoon E and Wise K D A multi-element monolithic mass flowmeter with on-chip CMOS readout electronics IEEE Solid-State Sensor and Actuator Workshop (Hilton-Head, SC, 1990) Technical digest pp 161-4

[21] Najafi N and Wise K D 1990 An organization and interface for sensor-driven semiconductor process control systems IEEE Trans. on Semiconductor Manufacturing vol 3 (New York; IEEE) pp 230-8

[22] Wise K D and Najafi K 1988 vLsI sensors in medicine VLSI In Medicine ed Norman G. Einspruch and Robert D Gold (New York: Academic)

[23] Fan L S, Tai Y C and Muller R S 1989 IC-processed electrostatic micromotors Sensors and Actuators 20 41-7

[24] Tang W C, Nguyen T C H and Howe R T 1989 Laterally-driven polysilicon resonant microstructures Sensors and Actuators 20 25-32

[25] Hart K and Slob A 1972 Integrated injection logic: a new approach to LSI IEEE J. Solid-State Circuits SC-7 346-51

[26] Kubo M, Masuda I, Miyata K and Ogiue K 1988 Perspective on BICMOS VLSI IEEE J. Solid-State Circuits SC-23 5-11

[27] Ziaie B, Gianchandani Y and Najafi K 1991 A highcurrent IrOx thin-film neuromuscular microstimulator 6th Int. Conf. on Solid-State Sensors and Actuators (San Francisco, 1991) unpublished

[28] Vittoz E A 1983 mos transistors operated in the lateral bipolar mode and their applications in CMOS technology IEEE J. Solid-State Circuits SC-18 2739

[29] Paramesvaran M, Ristic L, Chau K, Robinson A M and Allegretto $\mathrm{W}$ CMOS electrothermal microactuators IEEE Micro Electro Mechanical Systems Workshop (Napa, CA, 1990) Technical digest pp 128-31

[30] Paramesvaran M, Baltes H P and Robinson A M 1986 Polysilicon microbridge fabrication using standard CMOS technology IEEE Solid-State Sensor and Actuator Workshop (Hilton-Head, SC, 1986) Technical digest

[31] Brown R, Catron $\mathbf{K}$ and Wright $W$ Considerations in the high-volume production of hybrid pressure sensor modules for automotive applications IEEE Solid-State Sensor and Actuator Workshop (HiltonHead, SC, 1988) Technical digest pp $148-50$

[32] Mallon J, Bryzek J, Ramsey J, Tomblin G and Pourahmadi F Low-cost, high-volume packaging techniques for silicon sensors and actuators $I E E E$ Solid-State Sensor and Actuator Workshop (HiltonHead, SC, 1988) Technical digest pp 123-4

[33] Brown R B, Wu K C, Ghezzo M and Brown D M A CMOs process for high-temperature sensors and circuits IEEE Solid-State Sensor and Actuator Workshop (Hilton Head, 1988) Technical digest pp 117-8

[34] Spangler L J and Wise K D 1990 A bulk silicon sor process for active integrated sensors Sensors and Actuators A24 117-22

[35] Wallis G D and Pomerantz D I 1969 Field-assisted glass-metal sealing J. Appl. Phys. 40 3946-9

[36] Esashi M, Nakano A, Shoji S and Hebiguchi H 1990 Low-temperature silicon-to-silicon anodic bonding with intermediate low melting point glass Sensors and Actuators A21-3 931-4

[37] Field L A and Muller R S 1990 Fusing silicon wafers with low melting temperature glass Sensors and Actuators A21-3 935-8

[38] Akin T, Ziaie B and Najafi K RF telemetry powering 
and control of hermetically sealed integrated sensors and actuators IEEE Solid-State Sensor and Actuator Workshop (Hilton-Head, SC, 1990) Technical digest pp 145-8

[39] Najafi K, Ji J and Wise K D Multichannel intracortical recording microprobes: scaling limitations, device characteristics, and circuit encapsulation Proc., Transducers ' 87 , Int. Conf. on Solid-State Sensors and Actuators (Tokyo, Japan, 1987) (Tokyo: IEE Japan) pp 65-8

[40] Borky J M and Wise K D 1979 Integrated signal conditioning for silicon pressure sensors $I E E E$ Trans. on Electron Devices ED-26 1906-10

[41] Goodenough F 1985 Sensor ICs: processing, materials open factory doors Electronic Design (Tokyo: IEE Japan) pp 131-48

[42] Najafi K and Wise K D 1986 An implantable multielectrode array with on-chip signal processing IEEE J. Solid-State Circuits SC-21 1035-44

[43] Choi I H and Wise K D 1986 A silicon-thermopilebased infrared sensing array for use in automated manufacturing IEEE Trans. on Electron Devices ED-
$3372-9$

[44] Johnson C L, Wise K D and Schwank J·W 1988 A thin-film gas detector for semiconductor process gases IEEE Int. Electron Device Meeting Technical digest pp 662-5

[45] Yoon E and Wise K D 1989 A monolithic RMS-DC converter using planar diaphragm structures Int. Electron Device Meeting Technical digest pp 491-4

[46] Najafi N, Clayton K W, Baer W G, Najafi K and Wise $K \mathrm{D}$ An architecture and interface for VLSI sensors IEEE Solid-State Sensor and Actuator Workshop (Hilton-Head, SC, 1988) Technical digest pp 74-9

[47] Leuthold H and Rudolf F 1990 An AsIC for highresolution capacitive microaccelerometers Sensors and Actuators A21-3 278-81

[48] Hasckard M R 1990 An experiment in smart sensor design Sensors and Actuators A24 163-9

[49] Crary S and Zhang Y CAEMEMS: an integrated computer-aided engineering workbench for microelectro-mechanical-systems IEEE Micro Electro Mechanical Systems Workshop (Napa, CA, 1990) Technical digest pp 113-4 